\title{
Review \\ Equipment review: Cooling catheters to induce therapeutic hypothermia?
}

\author{
Kees H Polderman ${ }^{1}$ and Jeannie Callaghan ${ }^{2}$
}

\author{
${ }^{1}$ Department of Intensive Care, University Medical Center Utrecht, P.O. Box 85500, 3508 GA Utrecht, The Netherlands \\ ${ }^{2}$ ALSIUS Corporation, Laguna Canyon Road, Irvine, California 92618-3111, USA
}

Corresponding author: Kees H Polderman, k.polderman@tip.nl or k.polderman@umcutrecht.nl

Published: 9 November 2006

This article is online at http://ccforum.com/content/10/6/234

(c) 2006 BioMed Central Ltd
Critical Care 2006, 10:234 (doi:10.1186/cc5023)

\begin{abstract}
There is growing acceptance within the medical community of induced (therapeutic) hypothermia as a tool to achieve neuroprotection and/or cardioprotection. Although much work remains to be done in identifying those clinical situations in which hypothermia can be effective, there is now sufficient evidence to regard it as a standard of care, at least for some indications such as selected patients with postanoxic encephalopathy. Thus, attention is now partly shifting from assessment of the clinical evidence of efficacy to technical and implementation issues. This review provides a list of criteria by which cooling devices can be judged, and specifically it discusses one of the new cooling devices: the Alsius CoolGard $3000^{\circledR}$ device and CoolLine ${ }^{\circledR}$ catheter. General aspects and advantages/disadvantages of surface versus core cooling are discussed, as are potential side effects, device-specific pros and cons, and cost-effectiveness issues. In addition, the current state of the evidence for use of induced hypothermia for various indications is briefly reviewed.
\end{abstract}

This article is part of the equipment reviews section, edited by Martin Chapman, David Gattas and Ganesh Suntharalingam.

The Section Editors contributed the abstract and introduction of this article

\section{Introduction}

At its conception, the purpose of this section was to explore new technologies by combining development details from industry with an independent reflection from the scientific community. This is no less so for the paired articles that follow.

Intravascular cooling catheters are among a range of proliferating technologies for use in temperature control. The release of such products into the market is problematic, especially given that the position of therapeutic hypothermia as a tool is still being defined. One area in which there is some clarity is in the case of postanoxic brain injury. The trials that provided us with this confidence used basic, old fashioned techniques of cooling, but they still yielded those vital risk reductions. We all have a responsibility to weigh cost against efficacy; for this technology, efficacy means both achieving the stated goals of the device and improving our patients' health. The industry may have given us evidence of the former but evidence of the latter remains to be reported. If there were greater potential clinical gain, then perhaps the costs could be considered acceptable. Other indications may soon be with us, for example in the next edition of the Brain Trauma Foundation guidelines for Traumatic Brain Injury, or for strict fever control for patients with stroke or subarachnoid hemorrhage. We can but wait.

\section{Technology questionnaire}

Jeannie Callaghan

\section{What is the science underlying the technology?}

Patients successfully resuscitated after cardiac arrest mainly receive supportive care, with only $5-30 \%$ of patients surviving to hospital discharge. Cardiac arrest survivors suffer from ischemic brain injury, leading to poor neurologic outcomes and death. Preliminary human trials investigating comatose survivors of cardiac arrest suggest favorable patient outcomes with initiation of therapeutic hypothermia.

$\mathrm{Cl}=$ confidence interval; $\mathrm{ICU}=$ intensive care unit; $\mathrm{OR}=$ odds ratio; $\mathrm{RCT}=$ randomized controlled trial. 
What are the primary indications for its use?

All ALSIUS products have the CE mark for general temperature management.

What are the common secondary indications for its use? Common secondary indications include reduction in fever in the neurologic intensive care unit (ICU), aneurysm surgery, emergency applications, and cardiac arrest.

\section{What are the efficacy data to support its use, including data over an existing gold standard, if appropriate?}

Two prospective randomized trials were published in 2002, which compared mild hypothermia $\left(32-32^{\circ} \mathrm{C}\right)$ with normothermia in comatose survivors of out-of-hospital cardiac arrest. One of the studies was conducted in five European countries [1] and the other took place in four hospitals in Melbourne, Australia [2].

\section{Are there any appropriate impact data available on the following: outcome, therapy, clinical behavior?}

The European study [1] showed that cooling to $32-34^{\circ} \mathrm{C}$ for 24 hours decreased the risk for death (odds ratio [OR] 0.74 , 95\% confidence interval $[\mathrm{Cl}]$ 0.58-0.95) and increased the likelihood of good neurologic recovery (OR 1.40, 95\% Cl
1.08-1.81). The Australian study [2] showed that cooling patients to $32-34^{\circ} \mathrm{C}$ for 12 hours increased the likelihood of good neurologic recovery (OR 2.65, 95\% Cl 1.02-6.88).

\section{What are the costs of using the technology, both initial and ongoing?}

This varies by market. In the USA the CoolGard $3000^{\circledR}$ system retails at about US\$28,500 and the cost of heat exchange catheters ranges between US\$300 and US\$1170.

\section{Are there any special user or patient requirements for the safe and effective use of this technology?}

A temperature sensing Foley catheter should be placed if available; otherwise, rectal or tympanic temperatures should be used (in that order) until a bladder probe is available.

\section{What is the current status of this technology and, if it is not in widespread use, why not?}

There are more than 4500 patients treated worldwide and 200 CoolGard $3000^{\circledR}$ systems installed globally. ALSIUS is the only cooling company to have completed a large randomized controlled trial (RCT) showing safety, efficacy, and nursing time in the neurologic ICU.

\section{Equipment review}

Kees H Polderman

Mild therapeutic hypothermia is being used with increasing frequency as a tool to prevent and/or mitigate various forms of neurologic injury. At this time the strongest evidence for its efficacy exists for various forms of postanoxic encephalopathy. Two RCTs published in 2002 [1,2] reported improved neurologic outcome in patients with postanoxic encephalopathy following witnessed cardiac arrest and an initial rhythm of ventricular fibrillation or ventricular tachycardia. This was followed in 2005 by the publication of two large multicentre RCTs in patients with neonatal asphyxia, which reported significant benefits of hypothermia treatment in selected infants with postanoxic injury $[3,4]$. Although the latter studies were performed in a different category of patients (newborns versus mostly middle-aged adults), the type of neurologic injury (global brain injury due to a transient period of oxygen deprivation) was analogous; the results of the studies in newborn infants thus provide support for the hypothesis that hypothermia can mitigate postanoxic brain injury in adults as well as in infants.

These observations have led to a recommendation by the European Resuscitation Council to use hypothermia following cardiac arrest if the initial rhythm was ventricular tachycardia or ventricular fibrillation, and to consider its use for other rhythms [5].

There are various other indications for which induced hypothermia can be used, with varying degrees of evidence
$[6,7]$. For example, it has been clearly demonstrated that induction of mild hypothermia can significantly decrease intracranial pressure in various types of brain injury, including traumatic brain injury, severe ischemic stroke, subarachnoid hemorrhage, and hepatic encephalopathy [6-8]. Many of these indications are controversial; for example, the evidence for the efficacy of hypothermia in improving neurologic outcome in traumatic brain injury is conflicting, with various single center studies reporting significant benefits in patients treated with hypothermia but one multicenter study failing to confirm these observations $[6,8]$. For indications such as stroke and subarachnoid hemorrhage preliminary data are promising, but no firm clinical evidence of neuroprotective effects is yet available.

A separate but closely related issue is the treatment and/or prevention of fever in patients with neurologic injury. Fever (defined as core temperature $\geq 38.5^{\circ} \mathrm{C}$ ) occurs frequently in these patients, and various clinical studies have shown that it is an independent predictor of adverse outcome [6,9]. Animal studies have shown that fever stimulates a number of destructive processes at the cellular level, thereby increasing the degree of neurologic injury $[6,10]$. Although no clinical RCTs have yet been performed to determine whether treatment of fever can indeed improve neurologic outcome in patients with neurologic injuries, more and more neurologic ICUs have implemented protocols for prompt and aggressive 
symptomatic treatment of fever, irrespective of its cause, based on the epidemiologic association of fever and adverse outcome and on the physiologic data listed above.

It is unclear how long the period following neurologic injury is during which neurologic outcome can still be influenced by treatments such as induced hypothermia. Clinical studies in patients with postanoxic encephalopathy $[2,6,7]$ have shown that benefits can be realized after periods of up to 12 hours. Indeed, the destructive processes triggered by an ischemic or traumatic episode continue for 48-72 hours, and can be reinitiated when new ischemic episodes occur. Such a new ischemic episode may be caused, for example, by an episode of intracranial hypertension [6,8]. Thus, in theory, the therapeutic 'window of opportunity' could be relatively large. However, based on observations in animals, it seems highly likely that the optimal effects are achieved when hypothermia is induced as quickly as possible - a concept summarized in the phrase 'time is brain'. In addition, a reliable maintenance phase with a minimum of temperature fluctuations is important, followed by a phase of slow and controlled rewarming because quick re-warming may re-trigger destructive processes at the cellular level [11].

Thus, to apply hypothermia successfully and/or maintain normothermia in a clinical setting, we need effective techniques to control temperature in a broad range of clinical situations. When inducing hypothermia, it is important to distinguish between the 'induction phase', when temperature is decreasing; the 'maintenance phase', when temperature is set and maintained at the desired level; and the re-warming phase, when the patient is slowly re-warmed in a controlled manner. A number of characteristics of an ideal temperature control device can be identified. First, it should enable high speed of induction with little or no 'overshoot' (decrease of temperature below the set level). It should enable reliable maintenance of temperature at the desired level within a narrow range, with few fluctuations above or below the set temperature, for prolonged periods of time. Slow and controlled re-warming to normothermia should be achievable. The device should allow reliable induction and maintenance of normothermia in febrile patients over prolonged periods of time (this can be significantly more difficult than maintaining hypothermia, because patients' physiologic mechanisms to raise temperature are less impaired than in hypothermic patients [11]). It should have a favorable profile with respect to both device-specific and general side effects. Finally, it should induce an acceptable amount of workload for the medical, nursing and technical staff; also, purchase and maintenance costs of the cooling device and disposables should be acceptable. Every cooling device should be judged according to these criteria.

Various cooling systems are currently available, each with specific advantages and disadvantages [12]. These can be roughly divided into invasive methods and noninvasive methods, with infusion of cold fluid as a separate technique that can be used as an accessory tool in the induction phase of hypothermia [13]. This review covers the properties of one of these cooling devices, namely the CoolGard $3000^{\circledR}$ system (ALSIUS Corporation). This core cooling device uses an indwelling central venous catheter (the CoolLine ${ }^{\circledR}$ catheter), which can be placed in the femoral vein (larger catheter with three cooling balloons) or the subclavian or jugular vein (smaller catheter with two cooling balloons). Sterile saline is refrigerated (to a minimum temperature of $4-5^{\circ} \mathrm{C}$ ) in the external device (the CoolGard $3000^{\circledR}$ ) and then pumped through the balloons coaxially mounted on the catheter, enabling direct cooling of the blood. The catheter contains a temperature probe enabling a 'closed loop' temperature control system; the temperature is set at the desired level (the range of the device is $28-38^{\circ} \mathrm{C}$, which may vary according to the installed software), after which the device cools the patient down to this level by decreasing or increasing the temperature of the circulating saline. The core temperature is then maintained at the desired level for as long as the attending physician deems necessary. The catheter also has two ports for central venous access, which can be used to administer medication and/or for blood sampling.

So, how does the CoolGard $3000^{\circledR}$ measure up when assessed according to the standards for the ideal/effective cooling device listed above? Experience with the device is still relatively limited, but initial reports (albeit in small numbers of patients) suggest that it is an effective and reasonably quick method for inducing hypothermia. Maximum cooling rates in the published studies have averaged at around $2-2.5^{\circ} \mathrm{C} /$ hour (for review $[11,12]$ ). These reports also suggest that the device is highly reliable at maintaining the desired temperature within a narrow range $\left( \pm 0.5^{\circ} \mathrm{C}\right)$ once the target temperature has been achieved. In addition, use of the device appears to be less labor intensive than 'conventional' methods for cooling (ice packs, fans, dousing with alcohol or water, among other approaches). Results in treating fever in the ICU have been more mixed. As explained above, on physiologic grounds it is more difficult to maintain normothermia than hypothermia because the patients' physiologic (warming) responses are less suppressed. In addition, conventional methods to combat fever such as use of fever-reducing drugs are relatively ineffective in reducing so-called 'central' (neurogenic) fever. Diringer [14] compared cooling catheters with 'traditional' cooling methods and found that the CoolGard $3000^{\circledR}$ device was significantly more effective; however, although the overall fever burden was reduced, many patients still had episodes of prolonged hyperthermia in that study. This problem could possibly be overcome by use of larger (threeballoon) catheters. With regard to safety and side effects, apart from the risks of the catheter insertion procedure (see below), the side effects risks appear to be limited to those inherent in hypothermia treatment [11]. The incidence of shivering may be lower than with other devices. 
(Potential) drawbacks of the device include the required insertion procedure, with associated procedural risks and time lost before cooling can be initiated. This implies that, for the moment, use of this technique is limited to the hospital setting (emergency room or ICU), and so it cannot be used in the ambulance or in the field.

Regarding the procedural risks, initial studies suggest that the risk for mechanical complications is comparable to that with insertion of 'traditional' central lines; larger studies will be required to confirm this. In theory the risk for catheterrelated thrombus formation (which is inherent in any indwelling device [15]) could be increased; however, none of the studies performed thus far has reported an increased risk for thrombosis. Few data are available regarding longer indwelling times; the manufacturer currently recommends a guidewire catheter exchange of the CoolLine ${ }^{\circledR}$ if the device is used for more than 7 days.

The price of the device in Europe is comparable to that of other cooling devices; the (disposable) catheters are relatively expensive, at $€ 500-1000$ (approximately US\$625-1250). However, the physician and nursing workload is low, and so the device may be cost-effective if these factors are taken into account. Further studies will be required to address these and other issues, and to compare this device with other cooling catheters and with various surface cooling technologies.

\section{Competing interests}

$\mathrm{JC}$ is an employee of Alsius Co. Over the past few years KP has received restricted educational grants from several companies involved in manufacturing hypothermia devices; however, Alsius corporation is not amongst them.

\section{References}

1. The Hypothermia after Cardiac Arrest Study Group: Mild therapeutic hypothermia to improve the neurologic outcome after cardiac arrest. N Engl J Med 2002, 346:549-556.

2. Bernard SA, Gray TW, Buist MD, Jones BM, Silvester W, Gutteridge G, Smith K: Treatment of Comatose Survivors of Outof-Hospital Cardiac Arrest with Induced Hypothermia. N Engl $J$ Med 2002, 346:557-563.

3. Shankaran S, Laptook AR, Ehrenkranz RA, Tyson JE, McDonald SA, Donovan EF, Fanaroff AA, Poole WK, Wright LL, Higgins RD, et al.: Whole-body hypothermia for neonates with hypoxicischemic encephalopathy. N Engl J Med 2005, 353:15741584.

4. Gluckman PD, Wyatt JS, Azzopardi D, Ballard R, Edwards AD, Ferriero DM, Polin RA, Robertson CM, Thoresen M, Whitelaw A, et al:: Selective head cooling with mild systemic hypothermia after neonatal encephalopathy: multicentre randomised trial. Lancet 2005, 365:663-670.

5. Nolan JP, Deakin CD, Soar J, Bottiger BW, Smith G: European Resuscitation Council. European Resuscitation Council guidelines for resuscitation 2005. Section 4. Adult advanced life support. Resuscitation 2005, 67 Suppl 1:S39-S86.

6. Polderman $\mathrm{KH}$ : Application of therapeutic hypothermia in the ICU: opportunities and pitfalls of a promising treatment modality. Part 1: Indications and evidence. Intensive Care Med 2004, 30:556-575.

7. Bernard SA, Buist M: Induced hypothermia in critical care medicine: a review. Crit Care Med 2003, 31:2041-2051.
8. Polderman $\mathrm{KH}$, Ely $\mathrm{EW}$, Badr $\mathrm{AE}$, Girbes $\mathrm{ARJ}$ : Induced hypothermia in traumatic brain injury: considering the conflicting results of meta-analyses and moving forward. Intensive Care Med 2004, 30:1860-1864.

9. Hajat C, Hajat S, Sharma P: Effects of post-stroke pyrexia on stroke outcome: meta-analysis of studies on patients. Stroke 2000, 31:410-414.

10. Ginsberg MD, Busto R: Combating hyperthermia in acute stroke: a significant clinical concern. Stroke 1998, 29:529-534.

11. Polderman $\mathrm{KH}$ : Application of therapeutic hypothermia in the intensive care unit. Opportunities and pitfalls of a promising treatment modality. Part 2: Practical aspects and side effects. Intensive Care Med 2004, 30:757-769.

12. Polderman $\mathrm{KH}$ : Keeping a cool head: how to induce and maintain hypothermia. Crit Care Med 2004, 32:2558-2560.

13. Polderman KH, Rijnsburger ER, Peerdeman SM, Girbes ARJ: Induction of hypothermia using large volumes of ice-cold intravenous fluid. Crit Care Med 2005, 33:2744-2751.

14. Diringer MN, for the Neurocritical Care Fever Reduction Trial Group: Treatment of fever in the neuro intensive care unit with a catheter based heat exchange system. Crit Care Med 2004, 32:559-564.

15. Polderman $\mathrm{KH}$, Girbes ARJ: Central venous catheter use part 1: Mechanical complications. Intensive Care Med 2002, 28:1-17. 